\title{
An accelerating and rotating Planck-Hubble universe (A very simple model of quantum cosmology)
}

\author{
U. V. S. Seshavatharam ${ }^{1} \&$ S. Lakshminarayana ${ }^{2}$ \\ ${ }^{1}$ Honorary Faculty, I-SERVE, Survey no-42, Hitech city, Hyderabad-84, Telangana, India. \\ ${ }^{2}$ Department of Nuclear Physics, Andhra University, Visakhapatnam-03, AP, India \\ Emails: seshavatharam.uvs@gmail.com (and)lnsrirama@gmail.com \\ Orcid numbers : 0000-0002-1695-6037 (and) 0000-0002-8923-772X
}

\begin{abstract}
With reference to Planck scale, increasing support for large scale cosmic anisotropy and preferred directions and by considering an increasing ratio of Hubble parameter to angular velocity, right from the beginning of Planck scale, we make an attempt to estimate ordinary matter density ratio, dark matter density ratio, mass, radius, temperature, age and expansion velocity (from and about the baby universe in all directions). We would like suggest that, from the beginning of Planck scale, 1) Dark matter can be considered as a kind of cosmic foam responsible for formation of galaxies. 2) Cosmic angular velocity decreases with square of the decreasing cosmic temperature. 3) Increasing ratio of Hubble parameter to angular velocity plays a crucial role in estimating increasing cosmic expansion velocity and decreasing density ratios of dark matter and ordinary matter. 4) There is no need to consider dark energy for understanding cosmic acceleration.
\end{abstract}

Keywords: Planck scale; quantum cosmology; critical density; ordinary matter; dark matter; expansion velocity; angular velocity; Hubble's law;

PACS Nos: 04.20.-q, 04.60.-m, 04.50.Kd, , 98.80.Qc, 98.80.Bp, 98.80.Es

\section{Introduction}

According to current notion of modern cosmology, if the known laws of physics are extrapolated to the highest density regime, the result is a singularity which is typically associated with the Big Bang [1,2]. Unfortunate thing is that, pre or post conditions and parameters of big bang physics are absolutely unknown. In this critical scenario, in a quantitative approach, it may not be wrong to consider a 'growing' or 'evolving' phase of 'Planck scale'. Even though massive nature is unclear - with known physical laws, Planck scale can be assigned with certain 'mass', certain 'radius', certain 'volume', certain 'density', certain 'temperature' and certain 'pressure' etc. Clearly speaking, Planck mass can be considered as a characteristic massive seed of the evolving universe and big bang can be replaced with an evolving Planck ball. Planck mass can be called as the 'baby universe'. Thus, by replacing 'big bang' with a growing Planck ball, in a hypothetical approach, an evolving model of quantum cosmology can be developed $[3,4]$. Since Planck scale is associated with Quantum theory and 'spin' is a basic property of quantum mechanics, it may not be wrong to consider a growing and rotating model [5-13] of a Planck ball from and about the baby universe in all directions. Center of the growing universe seems to depend on the location of the assumed Planck seed under consideration.

\section{Nomenclatures, assumptions and basic relations}

\subsection{Nomenclatures}

1) $H_{t}=$ Hubble parameter and $H_{p l}=$ Planck scale Hubble parameter.

2) $\left(\rho_{c} c^{2}\right)_{t} \cong\left(3 H_{t}^{2} c^{2} / 8 \pi G\right)=$ Critical energy density.

3) $\left(\Omega_{O M}\right)_{t}=$ Ratio of ordinary matter density to critical density.

4) $\left(\Omega_{D M}\right)_{t}=$ Ratio of dark matter density to critical density. 
5) $\omega_{t}=$ Cosmic angular velocity and $\omega_{p l}=$ Planck scale angular velocity.

6) $\quad\left(V_{\exp }\right)_{t}=$ Cosmic expansion velocity from and about the baby universe.

7) $\quad\left(M_{O M}\right)_{t}=$ Cosmic ordinary mass content

8) $\quad\left(M_{D M}\right)_{t}=$ Cosmic dark matter content.

9) $\quad\left(M_{O M}+M_{D M}\right)_{t} \cong M_{t}=$ Total matter content $=$ Total mass of evolving Planck ball.

10) $R_{t}=$ Cosmic radius associated with $M_{t}=$ Radius of evolving Planck ball.

11) $T_{t}=$ Cosmic temperature.

12) $\gamma_{t}=$ Ratio of Hubble parameter to angular velocity.

13) $\left(d_{g}\right)_{t}=$ Galactic distance from and about the baby universe.

14) $\left(v_{g}\right)_{t}=$ Galactic receding speed from and about the baby universe.

Note-1: For the above symbols, subscript 0 denotes current value and subscript $p l$ denotes Planck scale value.

\subsection{Proposed assumptions}

With respect to our earlier publications $[14,15]$, in this paper we review the basic assumptions.

1) Ratio of Hubble parameter to angular velocity is, $\gamma_{t} \cong\left(\frac{H_{t}}{\omega_{t}}\right) \cong\left[1+\ln \left(\frac{H_{p l}}{H_{t}}\right)\right] \cong \sqrt{\frac{3 H_{t}^{2} c^{2}}{8 \pi G\left(a T_{t}^{4}\right)}}$.

2) $\left(V_{\text {exp }}\right)_{t} \cong R_{t} H_{t} \cong \gamma_{t}^{1 / 4} c$ can be considered as the cosmic expansion velocity.

3) Cosmic radius can be expressed with $R_{t} \cong \frac{2 G M_{t}}{c\left(V_{\text {exp }}\right)_{t}}$

4) Dark matter can be considered as a kind of cosmic foam responsible for formation of galaxies $[16,17,18]$.

\section{Note points:}

(1) Assumption 1 indicates the role of Planck scale in entire cosmic evolution. Using the number $\gamma_{t}$, density ratios of dark matter and ordinary matter can be studied.

(2) Assumption 2 can be considered as a simplified form of Hubble's law applied to the expanding universe as whole. Assumption 2 can be understood in terms of cosmic kinetic energy and temperature in the following way. During cosmic evolution,

a) Kinetic energy of matter is inversely proportional to cosmic temperature.

b) Based on assumption 1, cosmic temperature can be shown to be inversely proportional to $\sqrt{\gamma_{t}}$.

c) Hence, cosmic expansion velocity can be shown to be proportional to $\gamma_{t}^{1 / 4}$.

(3) Assumption 3 resembles Schwarzschild radius relation with denominator having two velocities- $c$ refers to initial expansion velocity and $\left(V_{\text {exp }}\right)_{t}$ time dependent increasing expansion velocity.

(4) Assumptions 2 and 3 help in connecting cosmic expansion velocity and total matter density.

(5) Assumption 4 may help in studying the nature of dark matter. 


\subsection{Role of the Planck scale in entire cosmic evolution}

We make an attempt to implement the 'Planck scale' in the entire cosmic evolution. We define the Planck scale Hubble parameter, $H_{p l} \cong \sqrt{\frac{c^{5}}{G \hbar}} \cong 1.854921 \times 10^{43} \mathrm{sec}^{-1}$. To proceed further, we assume that,

$$
\begin{gathered}
\left(\frac{H_{t}}{\omega_{t}}\right) \cong \gamma_{t} \cong\left[1+\ln \left(\frac{H_{p l}}{H_{t}}\right)\right] \cong \sqrt{\left(\frac{3 H_{t}^{2} c^{2}}{8 \pi G\left(a T_{t}^{4}\right)}\right)} \\
\frac{3 \omega_{t}^{2} c^{2}}{8 \pi G} \cong a T_{t}^{4} \quad \text { and } \quad \omega_{\mathrm{t}} \cong \sqrt{\frac{8 \pi G\left(a T_{t}^{4}\right)}{3 c^{2}}}
\end{gathered}
$$

Based on relation (1), if defined $H_{p l} \cong 1.854921 \times 10^{43} \mathrm{sec}^{-1}$, one can choose different values of $\gamma$ in between $\gamma_{p l} \cong 1$ and $\gamma_{0} \cong 141.2564$ where $H_{0} \cong 70 \mathrm{~km} / \mathrm{sec} / \mathrm{Mpc} \cong 2.26853 \times 10^{-18} \mathrm{sec}^{-1}[19-22]$. For each assumed value of $H_{t}$, one can get a corresponding $\gamma_{t}$ and all other physical parameters can be estimated.

For the Planck scale, $\gamma_{p l} \cong 1$ and $\omega_{p l} \cong \frac{H_{p l}}{\gamma_{p l}} \cong H_{p l} \cong 1.854921 \times 10^{43} \mathrm{rad} / \mathrm{sec}$.

For the current case, $\gamma_{0} \cong 141.2564$ and $\omega_{0} \cong \frac{H_{0}}{\gamma_{0}} \cong 1.606 \times 10^{-20} \mathrm{rad} / \mathrm{sec} \cong 5.068 \times 10^{-13} \mathrm{rad} /$ year. This value can be compared with other estimates [5-13].

In a simplified form, cosmic temperature can be expressed as,

$$
T_{t} \cong \frac{1}{\sqrt{\gamma_{t}}}\left(\frac{3 H_{t}^{2} c^{2}}{8 \pi G a}\right)^{\frac{1}{4}} \cong\left\{\frac{0.652632 \hbar \sqrt{\omega_{p l} \omega_{t}}}{k_{B}}\right\}
$$

\section{Estimating the trend of density ratios of ordinary matter, dark matter and total matter}

\subsection{Total matter density ratio}

With the help of defined $\left(\frac{H_{t}}{\omega_{t}}\right) \cong \gamma_{t} \cong\left[1+\ln \left(\frac{H_{p l}}{H_{t}}\right)\right]$ and with reference to the current observed values of $\left(\Omega_{O M}\right)_{0}$ and $\left(\Omega_{D M}\right)_{0}$, we are making an attempt to estimate the past values of $\left(\Omega_{O M}\right)_{t}$ and $\left(\Omega_{D M}\right)_{t}$. We hope that, at Planck scale, there exits ordinary matter as well dark matter in certain proportions.

$$
\text { Let, } \begin{aligned}
\left(M_{O M}+M_{D M}\right)_{t} \cong M_{t} \cong\left[\left(\Omega_{O M}\right)_{t}+\left(\Omega_{D M}\right)_{t}\right]\left(\frac{3 H_{t}^{2}}{8 \pi G}\right)\left(\frac{4 \pi}{3} R_{t}^{3}\right) \\
R_{t} \cong \frac{2 G M_{t}}{c\left(V_{\text {exp }}\right)_{t}} \cong \frac{2 G\left(M_{O M}+M_{D M}\right)_{t}}{c\left(V_{\text {exp }}\right)_{t}}
\end{aligned}
$$




$$
M_{t} \cong\left(\frac{c\left(V_{\exp }\right)_{t}^{2}}{2 G H_{t}}\right) \text { and }\left(\frac{2 G M_{t} H_{t}}{\left(V_{\exp }\right)_{t}^{2}}\right) \cong c
$$

Based on these relations, it is possible to show that,

$$
R_{t} \cong \frac{2 G\left(M_{O M}+M_{D M}\right)_{t}}{c\left(V_{\exp }\right)_{t}} \cong \sqrt{\frac{1}{\left(\Omega_{O M}+\Omega_{D M}\right)_{t}}}\left(\frac{\sqrt{c\left(V_{\exp }\right)_{t}}}{H_{t}}\right) \cong \frac{\left(V_{\exp }\right)_{t}}{H_{t}}
$$

Based on assumption 2 and relation (7),

$$
\left(\Omega_{O M}+\Omega_{D M}\right)_{t} \cong \frac{c}{\left(V_{\exp }\right)_{t}} \cong \frac{1}{\gamma_{t}^{1 / 4}}
$$

\subsection{Relation between dark matter density ratio and ordinary matter density ratio}

Unless we know the physical nature and properties of dark matter, it may not be possible to thoroughly analyze the density ratios of dark matter and ordinary matter. Keeping the current values of $\left(\Omega_{O M}\right)_{0}$ and $\left(\Omega_{D M}\right)_{0}$ in view and guessing that, there exists $\left(\Omega_{O M}\right)_{p l}$ and $\left(\Omega_{D M}\right)_{p l}$ at Planck scale, we propose the following very simple 'model' relation. With further study, other such kind of relations can be developed in a verifiable approach and percentages of dark matter and ordinary matter can be explored [16].

$$
\begin{gathered}
\frac{\left(\Omega_{D M}\right)_{t}}{\left(\Omega_{O M}\right)_{t}} \cong \gamma_{t}^{1 / 3} \\
\left(\Omega_{O M}\right)_{t} \cong\left[\left(1+\gamma_{t}^{1 / 3}\right) \gamma_{t}^{1 / 4}\right]^{-1} \\
\left(\Omega_{D M}\right)_{t} \cong\left[\left(1+\gamma_{t}^{-1 / 3}\right) \gamma_{t}^{1 / 4}\right]^{-1}
\end{gathered}
$$

See figure 1 plotted with relations (8), (10) and (11). Blue curve represents a decreasing trend of ordinary matter density ratio, black curve represents a decreasing trend of dark matter density ratio and green curve represents a decreasing trend of total matter density ratio.

Here, it is very importnt to note that, even though density ratios of ordinary matter and dark matter are assumed have a decreasing trend, their mass content can be shown to be increasing with incleasing cosmic radius and volume. In terms of $\left(\Omega_{O M}\right)_{t}$ and $\left(\Omega_{D M}\right)_{t}$,

$$
\begin{gathered}
\left(M_{O M}\right)_{t} \cong\left[\frac{\left(\Omega_{O M}\right)_{t}}{\left(\Omega_{O M}+\Omega_{D M}\right)_{t}}\right]\left(\frac{c\left(V_{\text {exp }}\right)_{t}^{2}}{2 G H_{t}}\right) \cong\left(\Omega_{O M}\right)_{t}\left(\frac{\left(V_{\text {exp }}\right)_{t}^{3}}{2 G H_{t}}\right) \\
\left(M_{D M}\right)_{t} \cong\left[\frac{\left(\Omega_{D M}\right)_{t}}{\left(\Omega_{O M}+\Omega_{D M}\right)_{t}}\right]\left(\frac{c\left(V_{\text {exp }}\right)_{t}^{2}}{2 G H_{t}}\right) \cong\left(\Omega_{D M}\right)_{t}\left(\frac{\left(V_{\text {exp }}\right)_{t}^{3}}{2 G H_{t}}\right)
\end{gathered}
$$

See table 1 for the current and Planck scale physical parameters. 
Figure 1: Decreasing trend of density ratios of ordinary matter, dark matter and total matter

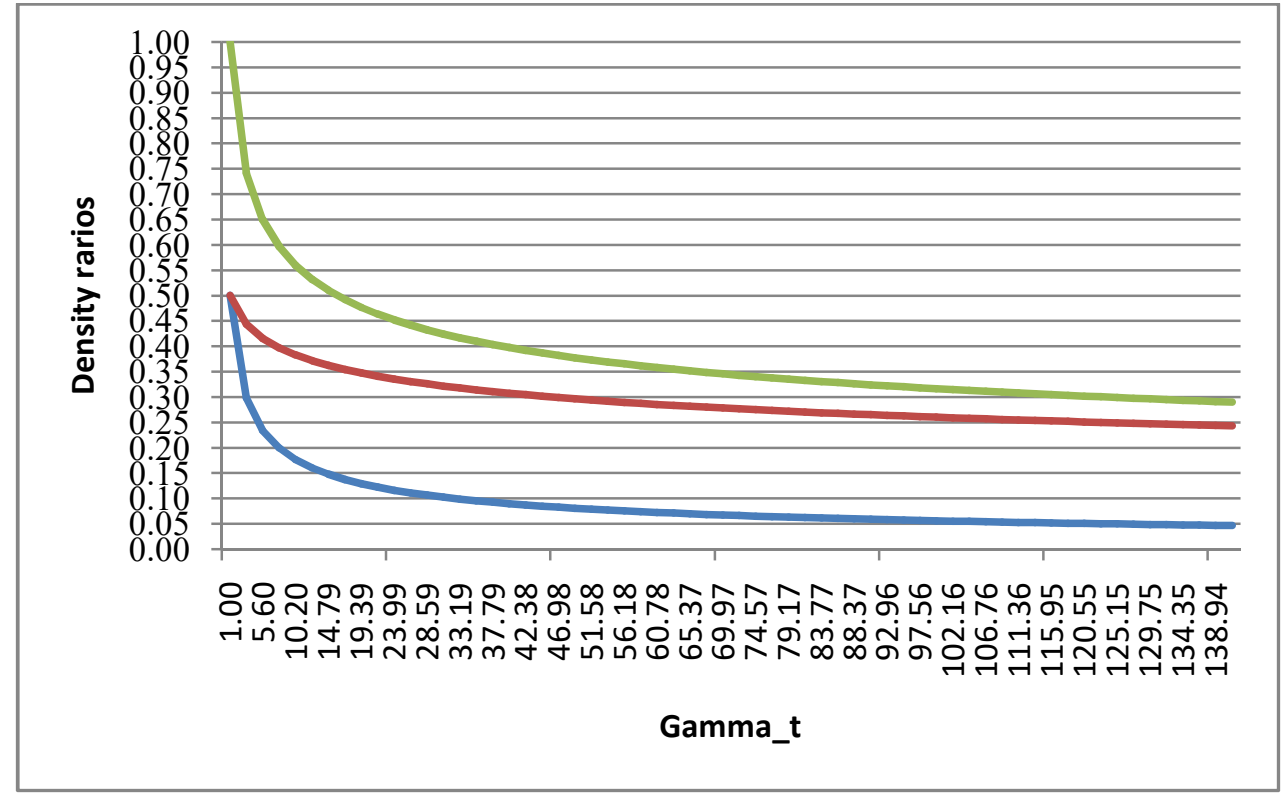

\begin{tabular}{|c|c|}
\hline \multicolumn{2}{|c|}{ Table 1: Current and Planck scale cosmic physical parameters } \\
\hline Current scale & Planck scale \\
\hline $\begin{array}{l}H_{0} \cong 70 \mathrm{~km} / \mathrm{sec} / \mathrm{Mpc} \\
\cong 2.26853 \times 10^{-18} \mathrm{sec}^{-1}\end{array}$ & $H_{p l} \cong \sqrt{\frac{c^{5}}{G \hbar}} \cong 1.855 \times 10^{43} \mathrm{sec}$ \\
\hline$\gamma_{0} \cong\left[1+\ln \left(\frac{H_{p l}}{H_{0}}\right)\right] \cong 141.2564$ & $\gamma_{p l} \cong\left[1+\ln \left(\frac{H_{p l}}{H_{p l}}\right)\right] \cong 1$ \\
\hline$\left(\Omega_{O M}\right)_{0} \cong 0.04673$ & $\left(\Omega_{O M}\right)_{p l} \cong 0.5$ \\
\hline$\left(\Omega_{D M}\right)_{0} \cong 0.2433$ & $\left(\Omega_{D M}\right)_{p l} \cong 0.5$ \\
\hline$T_{0} \cong\left(\frac{1}{\sqrt{\gamma_{0}}}\right)\left(\frac{3 H_{0}^{2} c^{2}}{8 \pi G a}\right)^{\frac{1}{4}} \cong 2.721 \mathrm{~K}$ & $\begin{array}{l}T_{p l} \cong\left(\frac{1}{\sqrt{\gamma_{p l}}}\right)\left(\frac{3 H_{p l}^{2} c^{2}}{8 \pi G a}\right)^{\frac{1}{4}} \\
\cong 9.247 \times 10^{31} \mathrm{~K}\end{array}$ \\
\hline $\begin{aligned} R_{0} & \cong \gamma_{t}^{1 / 4} \frac{c}{H_{0}} \cong 4.556 \times 10^{26} \mathrm{~m} \\
& \cong 14.772 \mathrm{Gpc}\end{aligned}$ & $R_{p l} \cong \gamma_{p l}^{1 / 4}\left(\frac{c}{H_{p l}}\right) \cong 1.616 \times 10^{-35} \mathrm{~m}$ \\
\hline$\left(V_{\text {exp }}\right)_{0} \cong R_{0} H_{0} \cong 3.4475 c$ & $\left(V_{\exp }\right)_{p l} \cong R_{p l} H_{p l} \cong c$ \\
\hline$\left(M_{O M}\right)_{0} \cong 1.7035 \times 10^{53} \mathrm{~kg}$ & $\left(M_{O M}\right)_{p l} \cong 5.441 \times 10^{-9} \mathrm{~kg}$ \\
\hline$\left(M_{D M}\right)_{0} \cong 8.87195 \times 10^{53} \mathrm{~kg}$ & $\left(M_{D M}\right)_{p l} \cong 5.441 \times 10^{-9} \mathrm{~kg}$ \\
\hline $\begin{array}{l}{\left[\left(M_{O M}\right)_{0}+\left(M_{D M}\right)_{0}\right]} \\
\cong M_{0} \cong 1.0575 \times 10^{54} \mathrm{~kg}\end{array}$ & $\begin{array}{l}{\left[\left(M_{O M}\right)_{p l}+\left(M_{D M}\right)_{p l}\right]} \\
\cong M_{p l} \cong 1.0882 \times 10^{-8} \mathrm{~kg}\end{array}$ \\
\hline Cosmic : & section-6) \\
\hline $\begin{array}{l}t_{0} \cong \frac{R_{0}}{\left[\left(\left(V_{\text {exp }}\right)_{0}+\left(V_{\text {exp }}\right)_{p l}\right) / 2\right]} \\
\cong 21.656 \text { BillionYears }\end{array}$ & $t_{p l} \cong 0$ \\
\hline
\end{tabular}




\section{Cosmic scale factor and red shift}

With reference to the proposed relations (1) and (3) and with reference to the current definitions of cosmic redshift and scale factor, it is possible to show that,

$$
\begin{gathered}
\left.\left(\frac{1}{a} \cong(z+1) \cong \frac{T_{t}}{T_{0}}\right) \cong \sqrt{\frac{\gamma_{0} H_{t}}{\gamma_{t} H_{0}} \cong \sqrt{\frac{\gamma_{0}}{\gamma_{t}}} \sqrt{\frac{H_{t}}{H_{0}}}}\right\} \\
\left.\cong \sqrt{\frac{\gamma_{0}}{\gamma_{t}}}\left\{\exp \left(\frac{\gamma_{0}-\gamma_{t}}{2}\right)\right\} \cong \sqrt{\left(\frac{\gamma_{0}}{\gamma_{t}}\right) \exp \left(\gamma_{0}-\gamma_{t}\right)}\right\} \\
z \cong \sqrt{\left(\frac{\gamma_{0}}{\gamma_{t}}\right) \exp \left(\gamma_{0}-\gamma_{t}\right)}-1
\end{gathered}
$$

\section{To estimate the current cosmic age}

From the beginning of cosmic evolution, based on the proposed cosmic expansion velocities, cosmic age can be approximated with the following relation.

$$
t \cong \frac{\left(R_{t}-R_{p l}\right)}{\left[\left(\left(V_{\exp }\right)_{t}+\left(V_{\exp }\right)_{p l}\right) / 2\right]}
$$

where $\left[\left(\left(V_{\exp }\right)_{t}+\left(V_{\exp }\right)_{p l}\right) / 2\right]$ can be considered as average expansion velocity.

For the current case, $t_{0} \cong 21.656$ BillionYears.

For a temperature of $3000 \mathrm{~K}$, it is possible to show that,

$$
\left.\begin{array}{l}
H_{3000 K} \cong 2.49 \times 10^{-12} \mathrm{sec}^{-1} \\
\gamma_{3000 K} \cong 1+\ln \left(\frac{H_{p l}}{H_{3000 K}}\right) \cong 127.34774 \\
Z_{3000 K} \cong \sqrt{\gamma_{3000 K}}-1 \cong 10.285 \\
z_{3000 K} \cong \sqrt{\frac{\gamma_{0}}{\gamma_{3000 K}} \exp \left(\gamma_{0}-\gamma_{3000 K}\right)}-1 \cong 1102.407
\end{array}\right\}
$$

Cosmic age corresponding to a temperature of $\mathrm{T}=3000 \mathrm{~K}$ can be estimated to be,

$$
t_{3000 K} \cong \frac{\left(R_{3000 K}-R_{p l}\right)}{\left[\left(\left(V_{\exp }\right)_{3000 K}+\left(V_{\exp }\right)_{p l}\right) / 2\right]} \cong 19613.65 \text { Years }
$$

This estimation is 19.37 times less than the current estimations and needs further study.

\section{Velocity and distance relation}

In all directions, from and about the hypothetical baby universe, current galactic receding speeds can be approximated with, 


$$
\left(v_{g}\right)_{0} \cong\left(\frac{\left(d_{g}\right)_{0}}{R_{0}}\right)\left(V_{\text {exp }}\right)_{0} \cong\left(\frac{\left(V_{\text {exp }}\right)_{0}}{R_{0}}\right)\left(d_{g}\right)_{0} \cong H_{0}\left(d_{g}\right)_{0}
$$

a) Relation (19) can be compared with currently believed Hubble's law for the current expanding universe.

b) For a distance of $\frac{c}{H_{0}}$, receding speed is $c$.

c) When, $\left(d_{g}\right)_{0} \rightarrow R_{0}, \quad\left(v_{g}\right)_{0} \cong H_{0} R_{0}$

\section{Results and Discussion}

\subsection{Cosmological constant problem}

With reference to proposed concepts, ratio of the Planck scale critical density to the current critical density is,

$$
\left(\frac{3 H_{p l}^{2} c^{2}}{8 \pi G}\right) \div\left(\frac{3 H_{0}^{2} c^{2}}{8 \pi G}\right) \cong\left(\frac{H_{p l}}{H_{0}}\right)^{2} \cong 6.685 \times 10^{121}
$$

This idea can be considered as a characteristic tool for constructing a model of 'quantum gravity' with cosmic evolution.

\subsection{Horizon problem}

If one is willing to consider the concept of 'matter causes the space-time to curve', 'horizon problem' can be understood. According to big bang model, during its evolution, as the universe is expanding, thermal radiation temperature decreases and matter content increases. As matter content increases, at any stage of evolution, it is possible to have an increasing radius of curvature. For the current case, $R_{0} \cong \gamma_{0}^{1 / 4} \frac{c}{H_{0}} \cong \frac{2 G M_{0}}{c\left(V_{\exp }\right)_{0}} \cong 14.772 \mathrm{Gpc}$ and there is no scope for 'causal disconnection' of distant visible matter.

\subsection{Cosmic inflation}

With our model, without considering 'inflation' concepts, starting from the Planck scale, it is possible to have a current cosmic radius of $14.772 \mathrm{Gpc}$ and it is consistent with current observations of $14.25 \mathrm{Gpc}$. Our estimated cosmic age corresponding to $2.7 \mathrm{~K}$ is around 21.66 billion years whereas big bang model estimation is 13.8 billion years. At lower time scales, our estimated cosmic age corresponding to $3000 \mathrm{~K}$ is around 19,614 years whereas big bang model estimation is $3,80,000$ years.

Point to be discussed in depth is, with big bang and inflation, after 3,80,000 years of evolution, cosmic temperature is $3000 \mathrm{~K}$ where as in our model, without big bang and inflation, after 19,614 years of cosmic evolution, temperature is $3000 \mathrm{~K}$. From this, it is very clear to say that, compared to big bang and inflation, in our model temperature drop is faster in the beginning and slower in the later stages. This can be considered as a hint for the observed large scale 'Isotropic' nature of CMBR.

\subsection{Cosmic acceleration and expansion velocity}


By considering a decreasing trend of ordinary matter and dark matter density, starting from the Planck scale, it is possible to get an expression for cosmic expansion velocity. It can be expressed as follows.

$$
\frac{\left(V_{\text {exp }}\right)_{t}}{c} \cong \gamma_{t}^{1 / 4} \cong\left[1+\ln \left(\frac{H_{p l}}{H_{t}}\right)\right]^{\frac{1}{4}} \cong\left(\frac{3 H_{t}^{2} c^{2}}{8 \pi G\left(a T_{t}^{4}\right)}\right)^{\frac{1}{8}} \cong \frac{1}{\left(\Omega_{O M}\right)_{t}+\left(\Omega_{D M}\right)_{t}}
$$

Based on this expression, for the Planck scale, $\left(V_{\exp }\right)_{p l} \cong c$ and for the current scale, $\left(V_{\text {exp }}\right)_{0} \cong 3.4475 c$. After 21.66 billion years of cosmic expansion, increment in expansion velocity seems to be $\left[\left(V_{\exp }\right)_{0}-\left(V_{\exp }\right)_{p l}\right] \cong 2.45 c$. See figure- 2 .

From figure 2 , it is very clear that, right from the beginning of cosmic evolution, cosmic expansion velocity seems to have an increasing trend. To some extent, this can be compared with currently believed cosmic acceleration concept [10-13]. Interesting point to be noted is that, expansion velocity seems to depend on $\frac{1}{\left(\Omega_{O M}\right)_{t}+\left(\Omega_{D M}\right)_{t}}$. In near future, if decrease in $\left(\Omega_{O M}\right)_{t}+\left(\Omega_{D M}\right)_{t}$ is found to be significant, one can expect 'acceleration' and if decrease in $\left(\Omega_{O M}\right)_{t}+\left(\Omega_{D M}\right)_{t}$ is found to be insignificant, one can expect cosmic 'constant rate of expansion'. It is for further study.

Figure 2: Increasing trend of cosmic expansion velocity

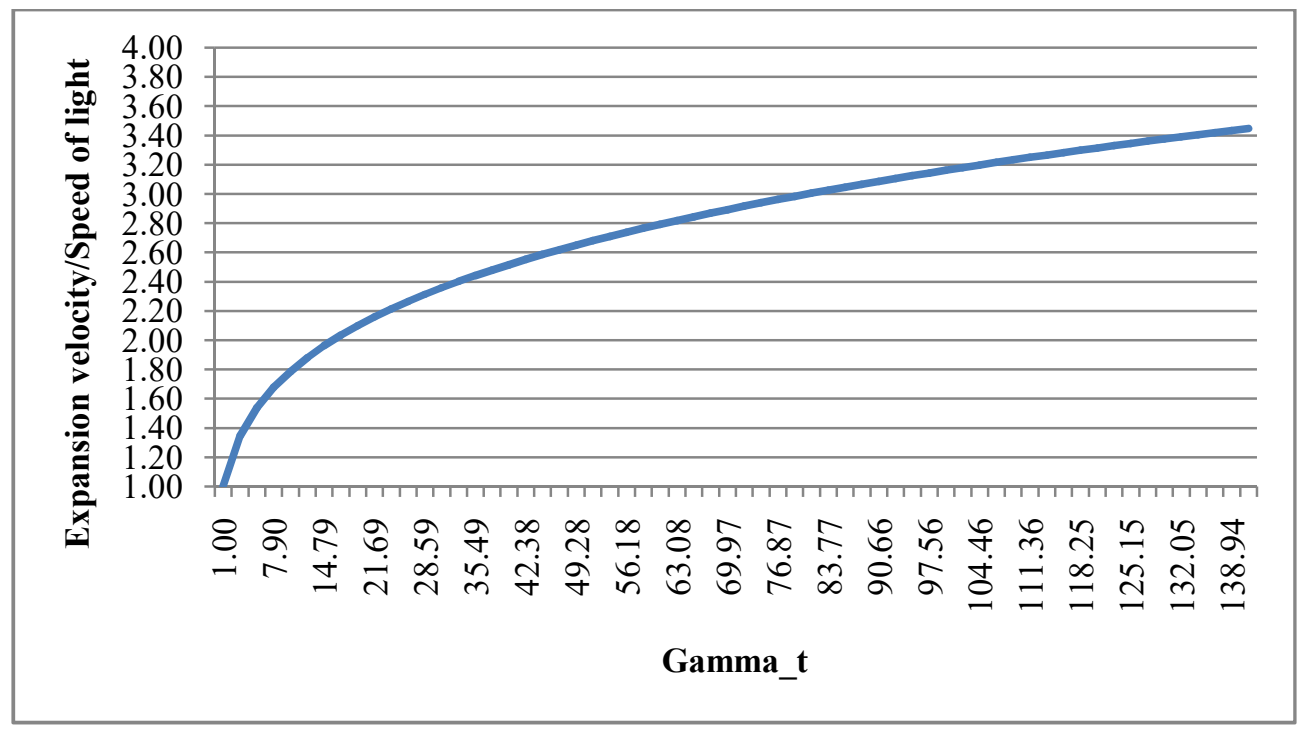

\subsection{Cosmic angular velocity}

With reference to our assumptions and relations, defined Planck scale angular velocity is $1.855 \times 10^{43} \mathrm{rad} / \mathrm{sec}$ and current angular velocity seems to be $\omega_{0} \cong 1.606 \times 10^{-20} \mathrm{rad} / \mathrm{sec} \cong 5.068 \times 10^{-13} \mathrm{rad} /$ year. This can be compared with other modern studies [5-13]. Here, interesting point to be noted is that, cosmic angular velocity decreases with decreasing cosmic temperature and decreasing Hubble parameter. The first experimental evidence of the Universe rotation was done by Birch [5], evidently. According to Birch, there appears to be strong evidence that the Universe is anisotropic on a large scale, producing position angle offsets in the polarization and brightness distributions of radio sources. These 
can probably be explained on the basis of a rotation of the Universe with an angular velocity of approximately $10^{-13} \mathrm{rad} /$ year. Observational effects of current cosmic rotation can be understood with the works of Obukhov [6], Godlowski [7] and Longo [8]. Now a days L.M. Chechin $[12,13]$ is seriously working on cosmic rotation.

\subsection{About dark matter}

Considering the very nature of Dark matter, new studies suggest that, a) Dark matter can be eliminated with emerging gravity concept [7]. b) Dark matter can be considered as a BoseEinstein condensate [8]. 3) Dark matter distribution can be understood through the distribution of intracluster light [9]. 3) Evidence for considering dark matter as a characteristic weakly interacting massive particle (WIMP) is geeting ruled out [23]. 4) In this critical situation, our proposal of considering dark matter as a kind of 'galactic foam' can be given some consideration. With further study, mystery of 'dark matter density ratio' can be explored with respect to different theoretically extended ideas of general theory of relativity or quantum cosmology.

\subsection{Cosmic anisotropy}

According to Shamik Ghosh et al [24] -The tantalizing possibility that the cosmological principle may be violated is indicated by many observations. The most prominent of these effects is the so-called Virgo alignment, which refers to a wide range of phenomena indicating a preferred direction pointing towards Virgo. The Square Kilometer Array has the capability to convincingly test several of these effects. These include the dipole anisotropy in radio polarization angles, the dipole in the number counts and sky brightness and in the polarized number counts and polarized flux. These observations may indicate that we need to go beyond the standard Big Bang cosmology. Alternatively they may be explained by preinflationary anisotropic and/or inhomogeneous modes. In either case, confirmation of this alignment effect is likely to revolutionize cosmology.

\section{Conclusions}

We would like appeal that, proposed decreasing trend of angular velocity, increasing trend of expanding speed (without dark energy), plotted graphs for understanding the smooth decreasing trend of density ratios of dark matter and ordinary matter, estimated noninflationary cosmic radius of $14.772 \mathrm{Gpc}$, early stage fast cooling and later stage slow cooling etc. seem to strengthen our proposed assumptions and semi empirical relations. With further study, big bang nucleosynthesis, inflation and cosmic time scales can be reviewed with respect to accelerating and rotating 'Planck-Hubble' universe.

\section{References}

[1] Gamow G Nature 162 p 680 (1948)

[2] Gamow G Phys Rev 74 p 505 (1948)

[3] Padmanabhan T Current Science 88 p 1057 (2005)

[4] Bojowald M Rep. Prog. Phys 78 p 023901 (2015)

[5] Birch P Nature Vol 298 p 451 (1982)

[6] Obukhov Y N Colloquium on Cosmic Rotation, Eds M. Scherfner, T. Chrobok and M. Shefaat (Wissenschaft und Technik Verlag: Berlin) p 23 (2000)

[7] Godlowski W International Journal of Modern Physics D 20 p 1643 (2011)

[8] Longo M Phys. Lett. B 699 p 224 (2011) 
[9] Godel K Rev. Mod. Phys. 21 p 447 (1949)

[10] Narlikar J V Mon. Not. R. Astr. Soc. 126 p 203 (1963)

[11] Hawking SW Mon. Not. R. Astr. Soc.142 p 129 (1969)

[12] Chechin LM Astron.Rep. 60 p 535 (2016)

[13] Chechin LM Gravit. Cosmol. 23 p 305 (2017)

[14] Seshavatharam U V S and Lakshminarayana S Physical Science International Journal 14(2) p 1 (2017)

[15] Seshavatharam U V S and Lakshminarayana S Physical Science International Journal 15(4): 1 2017

[16] Erik Verlinde SciPost Phy 2016 (2017)

[17] D.G. Levkov et al Phys. Rev. Lett 121, 151301 (2018)

[18] Mireia Montes and Ignacio Trujillo Mon.Not.Roy.Astron.Soc. 482 p 2838 (2019)

[19] Planck Collaboration: Planck 2015 Results XIII Cosmological Parameters (2015)

[20] Riess AG et al Astrophys.J 826 p 56 (2016)

[21] Riess AG et al Astron.J 116 p 1009 (1998)

[22] Perlmutter S et al Astrophys.J 517 p 565 (1999)

[23] https://www.forbes.com/sites/startswithabang/2019/02/22/the-wimp-miracle-is-dead-as-darkmatter-experiments-come-up-empty-again/\#18c618f26dbc

[24] Shamik Ghosh et al J. Astrophys. Astr. 37 p 25 (2016) 